\title{
PKD signaling and pancreatitis
}

\author{
Jingzhen Yuan $^{1} \cdot$ Stephen J. Pandol ${ }^{1,2}$
}

Received: 7 January 2016/Accepted: 25 January 2016/Published online: 15 February 2016

(C) Japanese Society of Gastroenterology 2016

\begin{abstract}
Background Acute pancreatitis is a serious medical disorder with no current therapies directed to the molecular pathogenesis of the disorder. Inflammation, inappropriate intracellular activation of digestive enzymes, and parenchymal acinar cell death by necrosis are the critical pathophysiologic processes of acute pancreatitis. Thus, it is necessary to elucidate the key molecular signals that mediate these pathobiologic processes and develop new therapeutic strategies to attenuate the appropriate signaling pathways in order to improve outcomes for this disease. A novel serine/threonine protein kinase D (PKD) family has emerged as key participants in signal transduction, and this family is increasingly being implicated in the regulation of multiple cellular functions and diseases.

Methods This review summarizes recent findings of our group and others regarding the signaling pathway and the biological roles of the PKD family in pancreatic acinar cells. In particular, we highlight our studies of the functions of PKD in several key pathobiologic processes associated with acute pancreatitis in experimental models.

Results Our findings reveal that PKD signaling is required for NF- $\kappa \mathrm{B}$ activation/inflammation, intracellular zymogen activation, and acinar cell necrosis in rodent experimental pancreatitis. Novel small-molecule PKD inhibitors attenuate the severity of pancreatitis in both in vitro and in vivo experimental models. Further, this
\end{abstract}

Jingzhen Yuan

jzyuan@ucla.edu

1 West Los Angeles VA Healthcare Center, UCLA/VA Greater Los Angeles Healthcare System, 11301 Wilshire Blvd, Bldg 258, Rm 340, Los Angeles, CA 90073, USA

2 Cedars-Sinai Medical Center, Los Angeles, CA, USA review emphasizes our latest advances in the therapeutic application of PKD inhibitors to experimental pancreatitis after the initiation of pancreatitis.

Conclusions These novel findings suggest that PKD signaling is a necessary modulator in key initiating pathobiologic processes of pancreatitis, and that it constitutes a novel therapeutic target for treatments of this disorder.

Keywords Protein kinase D · Pancreatitis - NF- $\kappa \mathrm{B}$ activation $\cdot$ Acinar cell necrosis $\cdot$ Zymogen activation

\section{Introduction}

Acute pancreatitis is a common and potentially lethal acute inflammatory disease. Alcohol abuse and gallstone disease are the major etiologic factors for pancreatitis, and account for the vast majority of the cases of this disease $[1,2]$. The hospitalization rate due to acute pancreatitis in the United States per year is approximately $300,000.20-30 \%$ of the cases develop into severe pancreatitis, usually due to pancreatic glandular necrosis [3]. However, management of acute pancreatitis is currently still limited to supportive care and treatment of complications.

The lack of clinical advances in the treatment of pancreatitis reflects the limited understanding of the early pathogenic mechanisms for this disease. A number of studies indicate that inflammation, parenchymal acinar cell death by necrosis, and inappropriate intracellular activation of digestive enzymes are the critical pathophysiologic processes for acute pancreatitis. However, the mechanisms and the key participants that mediate these processes in acute pancreatitis remain unclear. Our group has recently made significant advances in understanding the mechanisms associated with the key pathobiologic processes in 
pancreatitis. The present studies have revealed that a novel serine/threonine protein kinase, protein kinase D (PKD), plays a key role in regulating several early events in acute pancreatitis, which suggests that PKD may represent a novel molecular target for the treatment of acute pancreatitis.

\section{PKD structure and regulation in pancreatic acinar cells}

PKD is a serine/threonine protein kinase family within the $\mathrm{Ca}^{2+} /$ calmodulin-dependent protein kinase (CAMK) group. It has different structural, enzymatic, and regulatory properties from the protein kinase $\mathrm{C}$ (PKC) family members. $\mathrm{PKD} / \mathrm{PKD} 1$, also initially called $\mathrm{PKC} \mu$, is the founding and most extensively studied member of this kinase family, and has three isoforms: PKD/PKD1, PKD2, and PKD3 [4-7]. PKD family members have similar overall structures and primary amino acid sequences (Fig. 1). The most distinct characteristics of PKDs are the presence of a catalytic domain that is distantly related to $\mathrm{Ca}^{2+}$-regulated kinases and a pleckstrin homology $(\mathrm{PH})$ domain that regulates enzyme activity [8-13]. The N-terminal region of PKD also contains a cysteine-rich domain (CRD) consisting of a tandem repeat of cysteine-rich, zinc-fingerlike motifs, cys1 and cys 2 , which confer high binding affinity for phorbol esters and play a role in the regulation of catalytic kinase activity $[14,15]$. In unstimulated cells, PKD is in a state of low catalytic (kinase) activity maintained by autoinhibition mediated by the $\mathrm{NH}_{2}$-terminal domains.

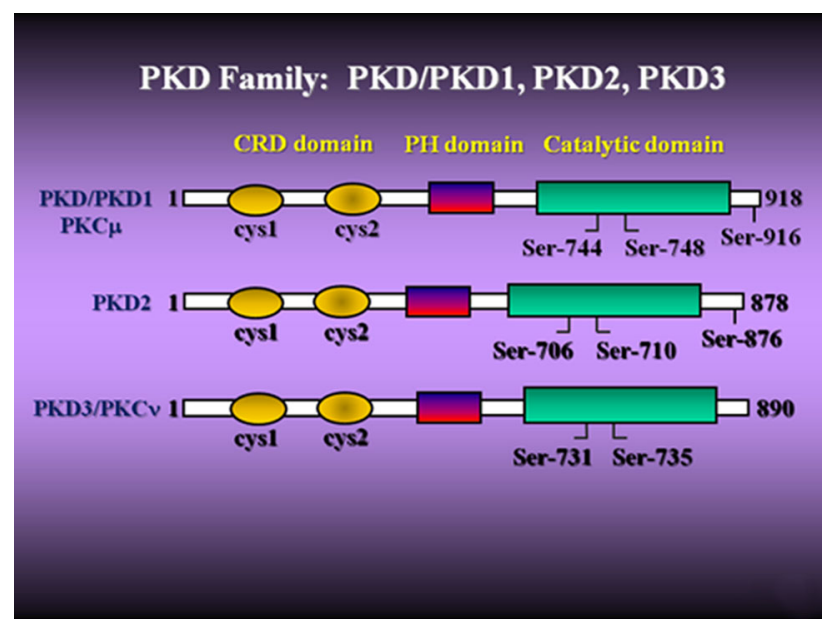

Fig. 1 The modular structure of PKD family isoforms. The PKD family comprises three serine kinases, PKD1, PKD2, and PKD3, with similar overall structures and primary amino acid sequences. All of them have a highly conserved $\mathrm{N}$-terminal regulatory domain containing two cysteine-rich DAG-binding (CRD) domains and an autoinhibitory pleckstrin homology $(\mathrm{PH})$ domain. Serine residues within the activation loop of PKDs that become phosphorylated via novel PKCs are indicated. Further details are provided in the text
PKD family members have recently emerged as major targets in the signal transduction pathways initiated by diacylglycerol (DAG) and PKC in a variety of cell types (reviewed in [11-13]), including pancreatic acinar cells $[16,17]$. PKD activation in intact cells is mediated mainly through PKCs. Earlier studies have demonstrated that PKC-dependent PKD phosphorylation at the activation loop serves as a direct "on/off" switch for catalytic activity (see $[12,18]$ for details). Activated PKD further autophosphorylates its Ser916 [12, 19]. In PKC-dependent $\mathrm{PKD}$ activation, the novel isoforms of PKC, including $\delta, \varepsilon$, $\eta$, and $\theta$, have been identified as upstream kinases of PKDs [20-23]. PKD family members are increasingly being implicated in the regulation of multiple cellular functions (review in [12]) in health and disease (review in [13]), such as protein secretion and Golgi function, phosphorylation of heat shock protein 27 [24] and histone deacetylase [25, 26], cell proliferation and apoptosis, oxidative stress and inflammation, and cardiac diseases and cancer [24-32]. However, the regulation and role of PKD family members in pancreatic acinar cells have not been studied.

Recently, our group and others explored the regulation of PKD isoforms in the major cells of exocrine pancreas, i.e., pancreatic acinar cells, from rats and mice [16, 17, 33, 34]. The studies showed that PKD/PKD1, the major PKD isoform in rat pancreatic acinar cells, is activated by phorbol esters and multiple pancreatic secretagogues, including cholecystokinin-8 (CCK-8 or CCK), the cholinergic agonist carbachol (CCh), and bombesin, but not by growth factors $[16,17,33,34]$. In isolated rat pancreatic acinar cells, CCK and $\mathrm{CCh}$ induced a dose-dependent rapid activation of PKD as measured by an in vitro kinase assay and by phosphorylation at the activation loop (Ser-744/748) or autophosphorylation site (Ser-916) [16, 17, 33]. The secretagoguestimulated PKD activation and phosphorylation occurs predominantly through a PKC-dependent pathway without involvement of Src/PI3K/MAPK/tyrosine phosphorylation $[16,17]$. Using isoform-specific inhibitory peptides for $\mathrm{PKC} \varepsilon$ and $\delta$ and genetic deletion of $\mathrm{PKC} \varepsilon$ and $\delta$ in pancreatic acinar cells, we further identified PKD as an early convergent point for PKC $\varepsilon$ and $\delta$ in the signaling pathways triggered by the pancreatic secretagogues [17].

As also seen for PKD/PKD1, we and others found that PKD3, the predominant isoform expressed in mouse pancreatic acinar cells, is also activated by gastrointestinal hormone or cholinergic stimulation [34]. PKD3 undergoes rapid membrane translocation, activation loop serine phosphorylation, and kinase activation after CCK or CCh stimulation. PKD3 phosphorylation in pancreatic acinar cells occurs via a $\mathrm{Ca}^{2+}$-independent but diacylglyceroland PKC-dependent mechanism.

It is unclear why the predominant PKD isoform expressed in the exocrine pancreas of mouse is different 
from that for the rat. Studying the regulatory mechanisms of PKD1 and PKD3 is, however, an important task, as evidence from the Human Protein Atlas project establishes that PKD1 and PKD3 are both moderately well expressed in human exocrine pancreas, and studies have shown that cholinergic stimulation can lead to PKD phosphorylation in human pancreatic acini [34].

\section{PKD mediates NF-אB activation and inflammation in pancreatitis}

PKD is a convergent point for $\mathrm{PKC} \delta$ and $\varepsilon$ in the signal pathways regulating NF- $\mathrm{\kappa B}$ activation in pancreatic acinar cells

Acute pancreatitis is an acute inflammatory process of the exocrine pancreas. There is emerging evidence that one of the early events in the development of acute pancreatitis is the initiation of an inflammatory response in the parenchymal cells of the pancreas [1,2,35-38]. The nuclear transcription factor NF- $\mathrm{KB}$ plays a critical role in the inflammatory response. NF- $\mathrm{KB}$ is one of the key signaling systems that mediate the production of pro-inflammatory cytokines, chemokines, immune receptors, and other inflammatory molecules, which subsequently lead to the severe systemic inflammatory complications of this disease [39-43]. In vivo studies in experimental pancreatitis demonstrated that NF- $\kappa B$ activation in acinar cells is one of the earliest events in pancreatitis, and that inhibiting NF- $\mathrm{KB}$ activation attenuates the severity of pancreatitis [39, 42]. Furthermore, the direct activation of NF- $\mathrm{KB}$ within the pancreas by adenovirus-mediated gene transfer is sufficient for the initiation of pancreatic and systemic inflammatory responses [43]. Therefore, elucidating the signaling mechanisms underlying NF- $\mathrm{\kappa B}$ activation is of critical importance for understanding the pathophysiology of pancreatitis.

PKCs were previously shown to stimulate NF- $\kappa B$ activation in various cell types and thus mediate cell survival as well as proinflammatory signaling [44-47]. Four PKC isoforms, $\alpha, \delta, \varepsilon$, and $\zeta$, have been detected in pancreatic acinar cells [48]. Although we demonstrated that the novel $\mathrm{PKC}$ isoforms $\mathrm{PKC} \delta$ and $\varepsilon$ are key regulators of NF- $\kappa \mathrm{B}$ activation induced by CCK [46], the downstream signaling targets of these PKCs within the NF- $\mathrm{BB}$ pathway in pancreatitis remain to be identified.

Consequently, we addressed the possibility that PKD lies downstream of PKCs in a signal transduction pathway activated by exocrine pancreas secretagogue $\mathrm{CCK}$ and cholinergic agonist $\mathrm{CCh}$ and mediates NF- $\mathrm{\kappa B}$ activation [17]. In this study, we initially observed that both CCK and CCh dose-dependently induced a rapid and striking activation of PKD in rat pancreatic acinar cells, as measured

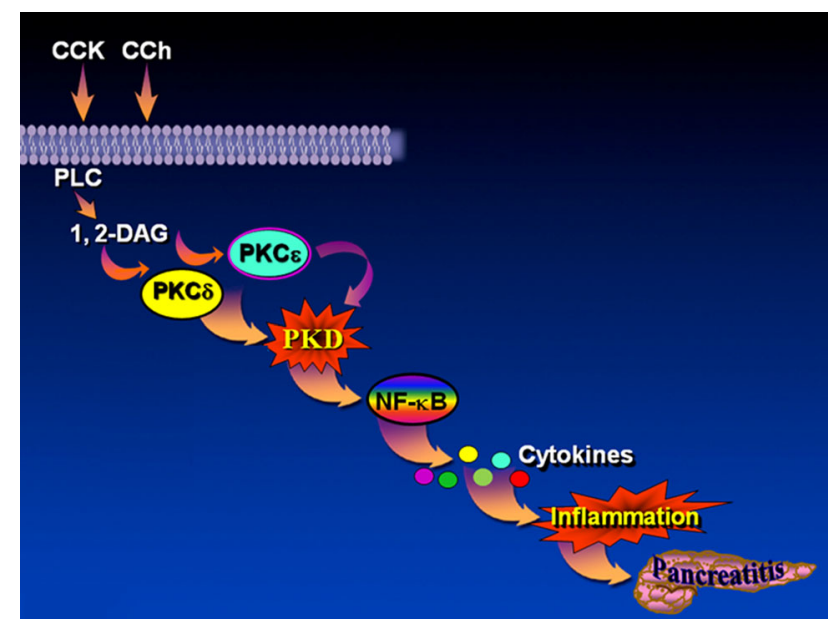

Fig. 2 PKD is a convergent point for $\operatorname{PKC} \delta$ and $\varepsilon$ in the signal pathways regulating NF- $\kappa \mathrm{B}$ activation in pancreatic acinar cells. PKD is regulated by signaling from both active $\mathrm{PKC} \delta$ and $\mathrm{PKC} \varepsilon$; active PKD further activates NF- $\kappa \mathrm{B}$ activation, resulting in inflammation and pancreatitis. Small-molecule PKD inhibitors can block the pathobiologic process. See the text for details

by in vitro kinase assay and by phosphorylation at the PKD activation loop (Ser744/748) or autophosphorylation site (Ser916). The phosphorylation and activation of PKD correlated with NF- $\mathrm{\kappa B}$ activity stimulated by $\mathrm{CCK}$ or $\mathrm{CCh}$, as measured by NF- $\mathrm{KB}$ DNA binding. We further compared the kinetics of PKD and NF- $\mathrm{KB}$ activation during cerulein-induced rat pancreatitis, and showed that both $\mathrm{PKD}$ and NF- $\mathrm{KB}$ activation were early events during acute pancreatitis and that their time courses of response were closely correlated. After that, we found that either inhibition of $\mathrm{PKC} \delta$ or $\varepsilon$ by isoform-specific inhibitory peptides, genetic deletion of PKC $\delta$ and $\varepsilon$ in pancreatic acinar cells, or knockdown of PKD using siRNAs in AR42J cells resulted in marked decreases in PKD and NF- $\mathrm{KB}$ activation stimulated by CCK or CCh. Conversely, overexpression of PKD1 resulted in augmentation of CCK- and CCh-stimulated NF- $\kappa \mathrm{B}$ activation [17].

Therefore, using a molecular/genetic approach, we identified PKD as a novel early convergent point for $\mathrm{PKC} \delta$ and $\varepsilon$ in the signaling pathways mediating NF- $\mathrm{KB}$ activation in pancreatitis (Fig. 2). These results demonstrated that PKD is a key downstream target of PKC $\delta$ and $\varepsilon$ in pancreatic acinar cells stimulated by two major secretagogues, $\mathrm{CCK}$ and $\mathrm{CCh}$, and that PKD is necessary for NF$\kappa \mathrm{B}$ activation in pancreatic acinar cells [17].

\section{Novel PKD inhibitors suppress NF-KB activation and inflammation in experimental models of pancreatitis}

The development and evaluation of specific PKD inhibitors have attracted intense interest in recent years due to the 
increasing recognition of multiple physiological and pathophysiological effects of PKD family members in humans. CRT0066101 and CID755673, two newly developed small-molecule pharmacological inhibitors that were isolated using a high-throughput screen to identify PKD family-specific inhibitors, have been recently reported for their anti-tumor-growth effects in pancreatic ductal adenocarcinoma and prostate cancer, respectively, by two research groups [31, 32].

In our studies, we investigated the effect of the two novel small-molecule PKD inhibitors on NF- $\mathrm{KB}$ activation using in vitro and in vivo experimental models of acute pancreatitis. Initially, we tested whether CID755673 and CRT0066101 are PKD-family-specific inhibitors. Pre-incubation of rat pancreatic acini with either CRT or CID for $1 \mathrm{~h}$ blocked agonist-induced PKD autophosphorylation at Ser916 without affecting PKC-dependent Ser744/748 phosphorylation (which was inhibited by the PKC inhibitor Go6983), demonstrating the specificity of these PKD inhibitors [33, 49]. Importantly, we found that NF-kBDNA binding activity in nuclear extracts, as measured by EMSA, was markedly suppressed by both CRT0066101 [33] and CID755673 (data to be published). The results support a role of PKD in NF- $\kappa B$ activation in pancreatic acinar cells.

Finally, we applied CID755673 in animal experimental pancreatitis to investigate whether PKD inhibition blocks $\mathrm{NF}-\kappa \mathrm{B}$ activation in vivo in acute pancreatitis. In agreement with our in vitro experimental results, cerulein, a CCK analog, induced a striking increase in activation of both PKD and NF- $\kappa B$ during the early stage of acute pancreatitis. Pre-treatment with CID755673 selectively suppressed cerulein-induced PKD kinase activation and autophosphorylation at Ser916 but did not affect PKC-dependent Ser744/748 phosphorylation [49]. The most exciting new finding was that the inhibition of PKD resulted in a significant decrease in NF- $\mathrm{KB}$ activation in CID755673-treated rats with pancreatitis (Yuan $\mathrm{J}$ et al., data to be published).

A hallmark of acute pancreatitis is the accumulation of neutrophils in the pancreas, which have been shown to modulate the severity of both the local changes as well as the systemic manifestation of pancreatitis. Depletion of neutrophils with anti-neutrophil serum leads to an attenuation of the severity of pancreatitis and associated lung injury $[1,37,38,50]$. Studies of NF- $\kappa B$ have demonstrated that it is activated in the pancreatic acinar cell early before the influx of inflammatory cells into the tissue. In addition to upregulating proinflammatory cytokines, NF- $\kappa \mathrm{B}$ can increase the effect of adhesion molecule ICAM-1 in the pancreas, which is involved in the systemic inflammatory response of acute pancreatitis through mediating neutrophil adhesion to the cells $[38,50]$.
Here, we examined the effect of PKD inhibition on pancreatic infiltration of inflammatory cells that are mostly neutrophils in pancreatitis [49]. Quantification of inflammatory cell infiltration was performed on H\&E-stained pancreatic tissue sections from late-stage pancreatitis (4 hourly cerulein injections). CID755673 treatment dramatically ameliorated the histological damage in ceruleininduced pancreatitis. One of the salient alterations in the pancreatic histological changes observed in the CID755673-pretreated animals was that cerulein-induced inflammatory cell infiltration was greatly $(70 \%)$ attenuated [49], which corresponded to the decreased NF- $\mathrm{KB}$ activation during the early stage of pancreatitis (Yuan $\mathrm{J}$ et al., data to be published).

Thus, using a pharmacological approach, we identified $\mathrm{PKD}$ as a critical mediator of NF- $\mathrm{KB}$ activation and inflammation, and demonstrated for the first time that PKD is required in NF- $\kappa B$ activation in an in vivo model of acute pancreatitis. The results suggest that novel smallmolecule PKD inhibitors have high potential as therapeutic agents to block/prevent severe pancreatitis in the early stage of the disease by suppressing NF- $\kappa \mathrm{B}$ activation (Fig. 2).

\section{PKD regulates zymogen activation and amylase secretion in pancreatitis}

Acinar cells elaborate a plethora of digestive enzymes that are synthesized and stored as inactive zymogen precursors to avoid autodigestion. Various in vitro and in vivo studies have established that the intra-acinar activation of zymogen is a key early event in the pathogenesis of pancreatitis. Activation of trypsinogen and other zymogens has been observed in the pancreatic homogenate as early as $10 \mathrm{~min}$ after supramaximal stimulation by cerulein in rats and increases over time [51-53]. The fact that other markers of pancreatitis, e.g., hyperamylasemia, pancreatic edema, and acinar cell vacuolization, can only be detected $30 \mathrm{~min}$ after supramaximal stimulation [52] strongly supports the paradigm that zymogen activation is the cause and not the result of pancreatitis. Similarly, in studies performed in vitro, supramaximal stimulation by CCK $\left(>10^{-10} \mathrm{~mol} / \mathrm{L}\right)$ results in zymogen activation and acinar cell injury [54, 55]. Moreover, pretreatment with protease inhibitors has been shown to reduce the severity of hyperstimulation pancreatitis in animals and to prevent acinar cell injury [5456]. These findings emphasize the role of zymogen activation in acinar cell injury caused by supramaximal secretagogue stimulation.

Evidence points to the pivotal role played by the novel isoforms of $\mathrm{PKC}, \mathrm{PKC}-\delta$ and $\mathrm{PKC}-\varepsilon$, in zymogen 
activation $[57,58]$. For the present study, we investigated whether the newly developed PKD inhibitor CRT0066101 would prevent zymogen activation in pancreatitis [33]. We pretreated isolated rat pancreatic acinar cells with CRT0066101 and then stimulated them for $60 \mathrm{~min}$ with high concentrations of CCK $(100 \mathrm{nM}), \mathrm{CCh},(1 \mathrm{mM})$, or bombesin $(10 \mu \mathrm{M})$ to induce the initial events of pancreatitis. PKD phosphorylation and activity as well as zymogen activation and amylase secretion were measured. Following specific and selective inhibition of secretagogue-induced PKD activation and autophosphorylation at Ser-916 by CRT0066101, secretagogue-induced zymogen activation was markedly reduced without affecting basal zymogen activation or secretion. Trypsin activities were reduced by 75,52 , and $53 \%$ of the maximum for CCK-, $\mathrm{CCh}$-, and bombesin-stimulated cells, respectively, which had been pretreated with CRT0066101. Chymotrypsin activities followed a similar pattern, with activity reductions of 75, 50, and $81 \%$ for CCK-, CCh-, and bombesinstimulated cells, respectively [33].

Another exciting result was that stimulation of amylase secretion by a pathologic dose of secretagogue (either $100 \mathrm{nM} \mathrm{CCK}, 1 \mathrm{mM} \mathrm{CCh}$, or $10 \mu \mathrm{M}$ bombesin) was significantly reduced following CRT0066101 pretreatment [33]. However, CRT0066101 pretreatment did not reduce amylase secretion induced by physiological concentrations of CCK $(0.1 \mathrm{nM})$, CCh $(1 \mu \mathrm{M})$, or bombesin $(10 \mathrm{nM})$. Interestingly, similar results were also achieved by another research group using molecular approaches in mouse pancreatic acinar cells [34]. Although PKD3 overexpression promoted amylase secretion, knockdown of PKD3 by RNA interference-induced gene silencing did not reduce the release of amylase mediated by a physiological dose of CCK [34]. The evidence that PKDs contribute to stimulated amylase release suggests that PKDs play a role in amylase release in pancreatitis.

We further studied the potential mechanism by which PKD mediates zymogen activation. The lysosomal hydrolase cathepsin B plays a role in intrapancreatic trypsinogen activation and the onset of acute pancreatitis [59, 60]. Therefore, we explored whether CRT0066101-dependent inhibition of PKD reduced cathepsin B activity [33]. We found that CCK-induced cathepsin B activity was inhibited by CRT0066101, indicating that PKD regulates cathepsinB-induced trypsinogen activation.

To summarize, our studies suggest that PKD is a potential mediator of zymogen activation and amylase secretion in pancreatitis. These events, together with NF$\kappa \mathrm{B}$ activation, are all early events that contribute to the pathologic process of acute pancreatitis. Our results further indicate that PKD is a potential target for therapy in the early stage of the disease.

\section{PKD promotes necrosis in pancreatitis by regulating cell death pathways}

\section{Cell death pathways in acute pancreatitis}

Apoptosis and necrosis are two major forms of acinar cell death in acute pancreatitis and are associated with specific morphological and biochemical features [61, 62]. Apoptosis is a tightly regulated process involving the caspase family of cysteine proteases, and manifests as chromatin condensation, nuclear shrinkage, and a reduction in cell volume but with intact plasma membranes, so there is no leakage of cellular contents into the extracellular environment. Conversely, necrosis leads to early plasma membrane permeabilization and rupture with release of intracellular constituents to the extracellular space. There is no sign of nuclear shrinkage $[1,61]$. Necrosis has been considered a passive, unregulated form of cell death; however, recent studies indicate that necrosis can occur in a tightly regulated fashion: so-called programmed necrosis [62-66]. Apoptosis is required for normal development and tissue homeostasis, and can also occur in pathologic conditions $[61,67,68]$. Necrosis exclusively occurs in pathologic situations and represents a severe cellular response to injury [61, 69]. Although these two types cell death have distinct morphologies, research findings [61, 70] suggest that the mechanisms underlying them are interrelated.

Understanding the regulation of the two death pathways in acute pancreatitis is important because the severity of acute pancreatitis in both humans and in experimental models correlates with the extent of necrosis [1]. In animal models of pancreatitis, the severity is also inversely correlated with apoptosis $[62,71-73]$. Therefore, revealing the key signaling molecules that determine the pattern of pancreatic acinar cell death (i.e., apoptosis versus necrosis) in pancreatitis will provide potential molecular targets for therapy in this disease.

\section{PKD regulates cell death pathways in pancreatitis}

We recently investigated the role and mechanisms of PKD in the regulation of necrosis in pancreatic acinar cells by using two novel small-molecule PKD inhibitors CID755673 and CRT0066101 and molecular approaches in both in vitro and in vivo experimental models of acute pancreatitis [49]. Our results demonstrated that PKD inhibition by either the chemical inhibitors or specific PKD/ PKD1 siRNAs attenuated necrosis while promoting apoptosis induced by pathological doses of CCK in pancreatic acinar cells. Conversely, upregulation of PKD expression in pancreatic acinar cells increased necrosis and decreased apoptosis. Further, in vivo treatment of animals with the 
PKD inhibitor CID755673 significantly ameliorated necrosis and the severity of cerulein-induced pancreatitis. These studies suggested that PKD activation promotes necrosis and inhibits apoptosis in both in vitro and in vivo models of pancreatitis.

We next explored the mechanisms by which PKD promoted necrosis. Apoptosis in pancreatic acinar cells is mediated mainly by activation of caspases. Importantly, it has been recognized that caspases not only mediate apoptosis but also protect from necrosis and decrease the severity of pancreatitis $[1,61,62,73]$. We found that upregulation of PKD expression prevented CCK/ceruleininduced activation of caspase- $9,-8$, and -3 and promoted necrosis. On the other hand, inhibition of PKD enhanced the activation of caspases in both in vitro and in vivo models of pancreatitis, resulting in decreased necrosis and severity of pancreatitis. Therefore, the pro-necrosis effect of PKD activation in pancreatitis is mediated through the inhibition of caspase activation [49].

$\mathrm{NF}-\kappa \mathrm{B}$-dependent anti-apoptotic gene products are an important class of endogenous protein inhibitors of caspase activation that include inhibitors of apoptotic proteins (IAPs) [1, 74-80]. Transcriptional activation of X-linked IAP (XIAP), survivin, and the anti-apoptotic FLICE-inhibitory protein (c-FLIP) through NF- $\kappa \mathrm{B}$ pathways has been demonstrated to be crucial in the regulation of cell death [1, 62, 74-80], including pancreatitis [1, 62]. Because we demonstrated that $\mathrm{PKD}$ regulates $\mathrm{NF}-\kappa \mathrm{B}$ activation in pancreatitis [17] and that NF- $\kappa B$ activation is significantly decreased by PKD inhibition during experimental pancreatitis [33], we examined the effect of PKD inhibition on these endogenous protein inhibitors of caspase activation. We found that PKD inhibition in ceruleininduced pancreatitis results in more rapid and greater decreases in the protein levels of XIAP, c-FLIPs, and survivin [49]. Using cell culture approaches, we showed that downregulation of PKD decreased protein levels of XIAP, c-FLIPs, and survivin, whereas upregulation of PKD expression enhanced protein levels of these IAPs, supporting a role for PKD in the regulation of IAPs. We also showed that inhibition of XIAP expression (as a representative of IAPs) significantly reduces necrosis while enhancing apoptosis and caspase activation, further supporting a mechanism by which PKD regulates cell death pathways through its effects on protein levels of IAPs.

A number of reports have indicated that the receptorinteracting protein kinase 1 (RIP1) is a key mediator of programmed necrosis [81-88], particularly in pancreatitis $[62,83]$. Caspases play a crucial role in the regulation of this key determinant of programmed necrosis. During death-domain-receptor-induced apoptosis, RIP1 is cleaved (i.e., inactivated) by caspase- 3 and -8. Therefore, RIP-dependent necrosis occurs especially in conditions in which caspases/apoptosis are inhibited or cannot be activated efficiently [62, 82, 85, 86]. Our results and the information described above prompted us to explore whether PKD regulates RIP1 in pancreatitis using pharmacological and genetic inhibition approaches [49]. Our studies indicate that the PKD/IAPs signaling pathway regulates the expression and inactivation of RIP1 in pancreatitis based on the following lines of experimental evidence: (1) pharmacological inhibition of PKD led to earlier and greater degradation/inactivation of RIP1 in cerulein-induced pancreatitis; (2) upregulation of PKD expression, which enhanced protein levels of XIAP and survivin, resulted in increased RIP1 production as well as decreased degradation; (3) downregulation of PKD with PKDsiRNAs, which decreased the protein levels of these IAPs, promoted RIP1 cleavage/inactivation; (4) genetic inhibition of XIAP expression markedly increased RIP1 cleavage/inactivation in the pancreatic acinar cells. These results indicate that PKD regulates RIP1 expression and inactivation through the IAPs [49].

In summary, our studies demonstrate that that PKD mediates necrosis in pancreatitis through mechanisms involving key cell death signals, including IAPs, caspases, and RIP1. PKD activation in pancreatitis increases the levels of anti-apoptotic proteins, XIAP, c-FLIPs, and survivin, which suppress caspase activation and inhibit apoptosis. Caspase inhibition also results in decreased cleavage/inactivation of RIP1, thus leading to increased necrosis and severity of pancreatitis. This cell necrotic death response is blocked when PKD is inhibited. PKD inhibition promotes apoptosis and decreases necrosis. Thus, PKD inhibition switches cell death away from necrosis and ameliorates the severity of pancreatitis. Our studies demonstrate that PKD is a key mediator of necrosis in acute pancreatitis and may represent a potential therapeutic target in acute pancreatitis [49] (Fig. 3).

\section{Therapeutic application of PKD inhibitors in experimental pancreatitis}

Although our studies have shown that pre-treatment with PKD inhibitor CRT0066101 or CID755673 significantly attenuates pancreatitis response, there is limited knowledge regarding the application of $\mathrm{PKD}$ inhibitors as therapeutic agents for pancreatitis in experimental animal models. Very recently, we evaluated the therapeutic effect of PKD inhibitor CID755673 on the severity of acute pancreatitis in experimental animal models when the CID755673 was given after the initiation of pancreatitis (Yuan $\mathbf{J}$ et al., data to be published).

Our results show that CID755673 treatment significantly decreased cerulein-induced serum amylase activities by $78 \%$ in the inhibitor-treated animals. Histological staining 


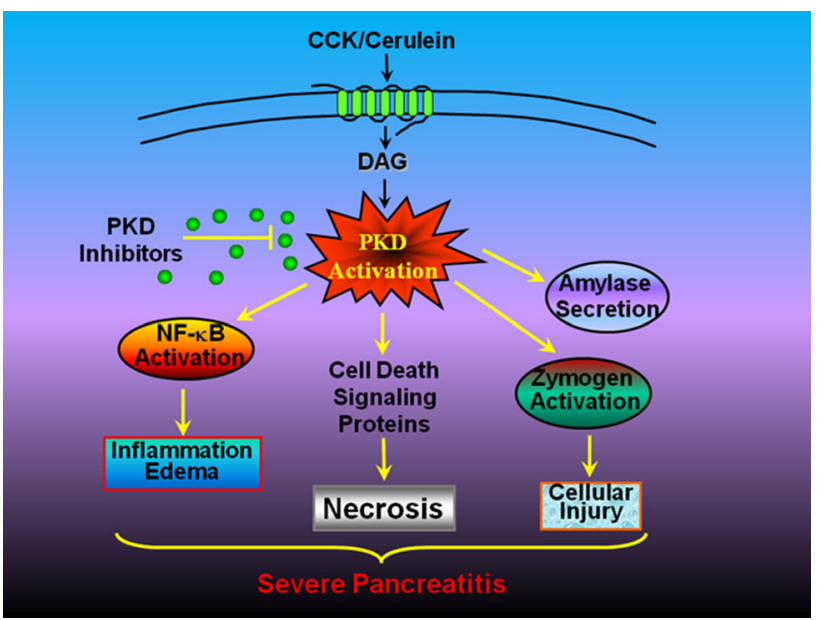

Fig. 3 A scheme summarizing the role of PKD in multiple pathological processes associated with pancreatitis. CCK/cerulein-induced PKD activation promotes necrosis in pancreatitis by regulating multiple cell death signaling proteins. Active PKD also mediates NF$\kappa \mathrm{B}$ activation and inflammatory response, zymogen activation, and amylase secretion. These pathologic responses can be blocked when PKD is inhibited by specific PKD inhibitors, resulting in amelioration of the severity of pancreatitis. All these effects indicate that PKD may represent a potential therapeutic target in pancreatitis. See the text for details

of the pancreatic tissue from either mice or rats shows that treatment of CID755673 significantly attenuates histopathologic damage in the pancreas. Cerulein-induced necrosis, inflammatory cell infiltration, and vacuole accumulation were reduced by 50-60\% in CID-treated rodents compared to vehicle-treated animals after 6 hourly (rats) or 7 hourly (mice) cerulein injections (Yuan J et al., data to be published).

These studies suggest that small-molecule inhibitors of PKD can be used to attenuate inflammation, necrosis, and the severity of acute pancreatitis when given after the development of the disease. Thus, these agents have the potential for development as therapeutic agents for the treatment of acute pancreatitis.

\section{Conclusion}

In conclusion, pancreatitis is a common and serious medical disorder, and novel molecularly targeted therapies are urgently needed. The studies discussed here established that PKD is a molecular signal that promotes the development of severe necrotizing pancreatitis through at least three mechanisms: (1) PKD plays a critical role in inflammatory response by mediating the activation of the key transcription factor NF- $\kappa \mathrm{B}$ in pancreatitis; (2) PKD mediates premature of zymogen and secretion of amylase, leading to pancreatic tissue injury; (3) PKD promotes pancreatic acinar cell necrosis by regulating several key death signaling proteins. Currently available small-molecule PKD inhibitors have been shown to have therapeutic effects in experimental models of acute pancreatitis in animals, attenuating inflammation and necrosis. Thus, PKD is potentially a valuable therapeutic target in pancreatitis. Potent and specific PKD inhibitors have the potential for development as therapeutic agents of acute pancreatitis (Fig. 3).

Acknowledgments This study was supported by a Department of Veterans Affairs Merits Grant and Southern California Research Center for Alcoholic Liver and Pancreatic Diseases Pilot Project Study Awards (NIH Grant P50-A11999) CA163200 and DK108314.

\section{References}

1. Pandol SJ, Saluja AK, Imrie CW, Banks PA. Acute pancreatitis: bench to the bedside. Gastroenterology. 2007;132:1127-51.

2. Pandol SJ, Raraty M. Pathobiology of alcoholic pancreatitis. Pancreatology. 2007;7:105-14.

3. Everhart JE, Ruhl CE. Burden of digestive diseases in the United States Part III: liver, biliary tract, and pancreas. Gastroenterology. 2009;136:1134-44.

4. Johannes FJ, Prestle J, Eis $\mathrm{S}$, et al. $\mathrm{PKCu}$ is a novel, atypical member of the protein kinase $\mathrm{C}$ family. J Biol Chem. 1994;269:6140-8.

5. Van Lint JV, Sinnett-Smith J, Rozengurt E. Expression and characterization of PKD, a phorbol ester and diacylglycerolstimulated serine protein kinase. J Biol Chem. 1995;270:1455-61.

6. Rey O, Yuan J, Rozengurt E. Intracellular redistribution of protein kinase D2 in response to G-protein-coupled receptor agonists. Biochem Biophys Res Commun. 2003;302:817-24.

7. Rey O, Yuan J, Young SH, Rozengurt E. Protein kinase CV/ protein kinase D3 nuclear localization, catalytic activation, and intracellular redistribution in response to $G$ protein-coupled receptor agonists. J Biol Chem. 2003;278:23773-85.

8. Rozengurt E, Sinnett-Smith J, Zugaza JL. Protein kinase D: a novel target for diacylglycerol and phorbol esters. Biochem Soc Trans. 1997;25:565-71.

9. Iglesias T, Rozengurt E. Protein kinase D activation by mutations within its pleckstrin homology domain. J Biol Chem. 1998;273: 410-6.

10. Waldron RT, Iglesias T, Rozengurt E. The pleckstrin homology domain of protein kinase D interacts preferentially with the eta isoform of protein kinase C. J Biol Chem. 1999;274:9224-30.

11. Guha S, Tanasanvimon S, Sinnett-Smith J, Rozengurt E. Role of protein kinase D signaling in pancreatic cancer. Biochem Pharmacol. 2010;80:1946-54.

12. Rozengurt E, Rey O, Waldron RT. Protein kinase D signaling. J Biol Chem. 2005;280:13205-8.

13. Rozengurt E. Protein kinase D signaling: multiple biological functions in health and disease. Physiology. 2011;26:23-33.

14. Iglesias T, Matthews S, Rozengurt E. Dissimilar phorbol ester binding properties of the individual cysteine-rich motifs of protein kinase D. FEBS Lett. 1998;437:19-23.

15. Iglesias T, Rozengurt E. Protein kinase D activation by deletion of its cysteinerich motifs. FEBS Lett. 1999;454:53-6.

16. Berna MJ, Hoffmann KM, Tapia JA, et al. CCK causes PKD1 activation in pancreatic acini by signaling through PKC-delta and PKC-independent pathways. Biochim Biophys Acta. 2007;1773:483-501. 
17. Yuan J, Lugea A, Zheng L, et al. Protein kinase D1 mediates NF$\kappa \mathrm{B}$ activation induced by cholecystokininand cholinergic signaling in pancreatic acinar cells. Am J Physiol Gastrointest Liver Physiol. 2008;295:G1190-201.

18. Jacamo R, Sinnett-Smith J, Rey O, et al. Sequential protein kinase $\mathrm{C}$ (PKC)-dependent and $\mathrm{PKC}$-independent protein kinase D catalytic activation via Gq-coupled receptors: differential regulation of activation loop $\operatorname{Ser}(744)$ and $\operatorname{Ser}(748)$ phosphorylation. J Biol Chem. 2008;283:12877-87.

19. Matthews SA, Rozengurt E, Cantrell D. Characterization of serine 916 as an in vivo autophosphorylation site for protein kinase D/protein kinase Cmu. J Biol Chem. 1999;274:26543-9.

20. Waldron RT, Rey O, Iglesias T, et al. Activation loop Ser744 and Ser748 in protein kinase D are transphosphorylated in vivo. J Biol Chem. 2001;276:32606-15.

21. Storz P, Doppler H, Toker A. Protein kinaseC delta selectively regulates protein kinase D- dependent activation of NF-kappaB in oxidative stress signaling. Mol Cell Biol. 2004;24:2614-26.

22. Waldron RT, Iglesias T, Rozengurt E. The pleckstrin homology domain of protein kinase D interacts preferentially with the eta isoform of protein kinase C. J Biol Chem. 1999;274:9224-30.

23. Yuan J, Bae D, Cantrell D, et al. Protein kinase D is a downstream target of protein kinase C theta. Biochem Biophys Res Commun. 2002;291:444-52.

24. Yuan J, Rozengurt E. PKD, PKD2, and p38 MAPK mediate Hsp27 serine-82 phosphorylation induced by neurotensin in pancreatic cancer PANC-1 cells. J Cell Biochem. 2008;103:648-62.

25. Matthews SA, Liu P, Spitaler M, et al. Essential role for protein kinase D family kinases in the regulation of class II histone deacetylases in B lymphocytes. Mol Cell Biol. 2006;26:1569-77.

26. Sinnett-Smith J, Ni Y, Wang J, et al. Protein kinase D1 mediates class IIa histone deacetylase phosphorylation and nuclear extrusion in intestinal epithelial cells: role in mitogenic signaling. Am J Physiol Cell Physiol. 2014;306:C961-71.

27. Storz P, Doppler H, Toker A. Protein kinase Cdelta selectively regulates protein kinase D-dependent activation of NF-kappaB in oxidative stress signaling. Mol Cell Biol. 2004;24:2614-26.

28. Chiu TT, Leung WY, Moyer MP, et al. Protein kinase D2 mediates lysophosphatidic acid-induced interleukin 8 production in nontransformed human colonic epithelial cells through NFkappaB. Am J Physiol Cell Physiol. 2007;292:C767-77.

29. Trauzold A, Schmiedel S, Sipos B, et al. PKCmu prevents CD95mediated apoptosis and enhances proliferation in pancreatic tumour cells. Oncogene. 2003;22:8939-47.

30. Fielitz J, Kim MS, Shelton JM, et al. Requirement of protein kinase D1 for pathological cardiac remodeling. Proc Natl Acad Sci USA. 2008;105:3059-63.

31. Harikumar KB, Kunnumakkara AB, Ochi N, et al. A novel small molecule inhibitor of protein kinase D blocks pancreatic cancer growth in vitro and in vivo. Mol Cancer Ther. 2010;9:1136-46.

32. Sharlow ER, Giridhar KV, LaValle CR, et al. Potent and selective disruption of protein kinase $\mathrm{D}$ functionality by a Benzoxoloazepinolone. J Biol Chem. 2008;283:33516-26.

33. Thrower EC, Yuan J, Usmani A, et al. A novel protein kinase D inhibitor attenuates early events of experimental pancreatitis in isolated rat acini. Am J Physiol Gastrointest Liver Physiol. 2011;300:G120-9.

34. Chen LA, Li J, Silva SR, et al. PKD3 is the predominant protein kinase $\mathrm{D}$ isoform in mouse exocrine pancreas and promotes hormone-induced amylase secretion. J Biol Chem. 2009;284: 2459-71.

35. Grady T, Liang P, Ernst SA, Logsdon CD. Chemokine gene expression in rat pancreatic acinar cells is an early event associated with acute pancreatitis. Gastroenterology. 1997;113: 1966-75.
36. Bhatia M, Brady M, Shokuhi S, Christmas S, Neoptolemos JP, Slavin J. Inflammatory mediators in acute pancreatitis. J Pathol. 2000;190:117-25.

37. Gukovskaya AS, Vaquero E, Zaninovic V, et al. Neutrophils and NADPH oxidase mediate intrapancreatic trypsin activation in murine experimental acute pancreatitis. Gastroenterology. 2002;122:974-84.

38. Frossard JL, Saluja A, Bhagat L, et al. The role of intercellular adhesion molecule 1 and neutrophils in acute pancreatitis and pancreatitis-associated lung injury. Gastroenterology. 1999;116: 694-701.

39. Gukovsky I, Gukovskaya AS, Blinman TA, et al. Early NFkappaB activation is associated with hormone-induced pancreatitis. Am J Physiol. 1998;275:G1402-14.

40. Vaquero E, Gukovsky I, Zaninovic V, et al. Localized pancreatic NF-kappaB activation and inflammatory response in taurocholate-induced pancreatitis. Am J Physiol Gastrointest Liver Physiol. 2001;280:G1197-208.

41. Tak PP, Firestein GS. NF-kappaB: a key role in inflammatory diseases. J Clin Invest. 1998;107:7-11.

42. Satoh A, Shimosegawa T, Fujita M, et al. Inhibition of nuclear factor-kappaB activation improves the survival of rats with taurocholate pancreatitis. Gut. 1999;44:253-8.

43. Chen X, Ji B, Han B, et al. NF-kappaB activation in pancreas induces pancreatic and systemic inflammatory response. Gastroenterology. 2002;122:448-57.

44. Koon HW, Zhao D, Zhan Y, et al. Substance P-stimulated interleukin-8 expression in human colonic epithelial cells involves protein kinase $\mathrm{C}$ delta activation. J Pharmacol Exp Ther. 2005;314:1393-400.

45. Page K, Li J, Zhou L, et al. Regulation of airway epithelial cell NF-kappa B-dependent gene expression by protein kinase C delta. J Immunol. 2003;170:5681-9.

46. Satoh A, Gukovskaya AS, Nieto JM, et al. PKC-delta and -epsilon regulate NF-kappaB activation induced by cholecystokinin and TNF-alpha in pancreatic acinar cells. Am J Physiol Gastrointest Liver Physiol. 2004;287:G582-91.

47. Zhao D, Zhan Y, Zeng H, et al. Neurotensin stimulates interleukin-8 expression through modulation of I kappa B alpha phosphorylation and p65 transcriptional activity: involvement of protein kinase C alpha. Mol Pharmacol. 2005;67:2025-31.

48. Bastani B, Yang L, Baldassare JJ, et al. Cellular distribution of isoforms of protein kinase $\mathrm{C}$ (PKC) in pancreatic acini. Biochim Biophys Acta. 1995;1269:307-15.

49. Yuan J, Liu Y, Tan T, et al. Protein kinase D regulates cell death pathways in experimental pancreatitis. Front Physiol. 2012;3:60.

50. Zaninovic V, Gukovskaya AS, Gukovsky I, et al. Cerulein upregulates ICAM-1 in pancreatic acinar cells, which mediates neutrophil adhesion to these cells. Am J Physiol Gastrointest Liver Physiol. 2000;279:G666-76.

51. Bialek R, Willemer S, Arnold R, Adler G. Evidence of intracellular activation of serine proteases in acute cerulein-induced pancreatitis in rats. Scand J Gastroenterol. 1991;26:190-6.

52. Grady T, Saluja A, Kaiser A, Steer M. Edema and intrapancreatic trypsinogen activation precede glutathione depletion during caerulein pancreatitis. Am J Physiol. 1996;271:G20-6.

53. Mithofer K, Fernandez-Del Castillo C, Rattner D, Warshaw AL. Subcellular kinetics of early trypsinogen activation in acute rodent pancreatitis. Am J Physiol. 1998;274:G71-9.

54. Grady T, Mah'Moud M, Otani T, et al. Zymogen proteolysis within the pancreatic acinar cell is associated with cellular injury. Am J Physiol. 1998;275:G1010-7.

55. Saluja AK, Bhagat L, Lee HS, et al. Secretagogue-induced digestive enzyme activation and cell injury in rat pancreatic acini. Am J Physiol. 1999;276:G835-42. 
56. Suzuki M, Isaji S, Stanten R, et al. Effect of protease inhibitor FUT-175 on acute hemorrhagic pancreatitis in mice. Int J Pancreatol. 1992;11:59-65.

57. Thrower EC, Osgood S, Shugrue CA, et al. The novel protein kinase $\mathrm{C}$ isoforms $-\delta$ and $-\varepsilon$ modulate caerulein-induced zymogen activation in pancreatic acinar cells. Am J Physiol Gastrointest Liver Physiol. 2008;294:G1344-53.

58. Thrower ECP, Wang JM, Cheriyan S, et al. Protein kinase C $\delta$ mediated processes in cholecystokinin-8-stimulated pancreatic acini. Pancreas. 2009;38:930-5.

59. Halangk W, Lerch MM, Brandt-Nedelev B, et al. Role of cathepsin $\mathrm{B}$ in intracellular trypsinogen activation and the onset of acute pancreatitis. J Clin Invest. 2000;106:773-81.

60. Halangk W, Lerch MM. Early events in acute pancreatitis. Clin Lab Med. 2005;25:1-15.

61. Gukovskaya AS, Pandol SJ. Cell death pathways in pancreatitis and pancreatic cancer. Pancreatology. 2004;4:567-86.

62. Mareninova OA, Sung KF, Hong P, et al. Cell death in pancreatitis: caspases protect from necrotizing pancreatitis. J Biol Chem. 2006;281:3370-81.

63. Moquin D, Chan FK. The molecular regulation of programmed necrotic cell injury. Trends Biochem Sci. 2010;35:434-41.

64. Krantic S, Mechawar N, Reix S, Quirion R. Apoptosis-inducing factor: a matter of neuron life and death. Prog Neurobiol. 2007;81:179-96.

65. Edinger AL, Thompson CB. Death by design: apoptosis, necrosis and autophagy. Curr Opin Cell Biol. 2004;16:663-9.

66. He S, Wang L, Miao L, et al. Receptor interacting protein kinase3 determines cellular necrotic response to TNF-alpha. Cell. 2009;137:1100-11.

67. Adams J. Ways of dying: multiple pathways to apoptosis. Genes Dev. 2003;17:2481-95.

68. Schultz DR, Harrington WJ Jr. Apoptosis: programmed cell death at a molecular level. Semin Arthritis Rheum. 2003;32:345-69.

69. Fiers W, Beyaert R, Declercq W, Vandenabeele P. More than one way to die: apoptosis, necrosis and reactive oxygen damage. Oncogene. 1999;18:7719-30.

70. Pandol SJ. Acute pancreatitis. Curr Opin Gastroenterol. 2006;22:481-6.

71. Kaiser AM, Saluja AK, Sengupta A, et al. Relationship between severity, necrosis, and apoptosis in five models of experimental acute pancreatitis. Am J Physiol. 1995;269:C1295-304.

72. Bhatia M, Wallig MA, Hofbauer B, et al. Induction of apoptosis in pancreatic acinar cells reduces the severity of acute pancreatitis. Biochem Biophys Res Commun. 1998;246:476-83.

73. Bhatia M. Apoptosis versus necrosis in acute pancreatitis. Am J Physiol Gasterointest Liver Physiol. 2004;286:G189-96.
74. Zou T, Rao JN, Guo X, et al. NF-kappaB-mediated IAP expression induces resistance of intestinal epithelial cells to apoptosis after polyamine depletion. Am J Physiol Cell Physiol. 2005;286:C1009-18.

75. Pahl HL. Activators and target genes of Rel/NF-kappaB transcription factors. Oncogene. 1999;18:6853-66.

76. Deveraux QL, Reed JC. IAP family proteins-suppressors of apoptosis. Genes Dev. 1999;13:239-52.

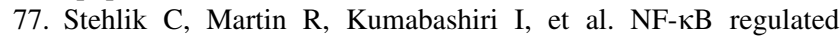
$\mathrm{X}$-chromosome-linked IAP gene expression protects endothelial cells from TNF- $\alpha$ induced apoptosis. J Exp Med. 1998;188: 211-6.

78. Kerbauy DM, Lesnikov V, Abbasi N, et al. NF-kappaB and FLIP in arsenic trioxide (ATO)-induced apoptosis in myelodysplastic syndromes (MDSs). Blood. 2005;106:3917-25.

79. Kawakami H, Tomita M, Matsuda T, et al. Transcriptional activation of survivin through the NF-kappaB pathway by human T-cell leukemia virus type I tax. Int J Cancer. 2005;115:967-74.

80. Bratton SB, Walker G, Srinivasula SM, et al. Recruitment, activation and retention of caspases- 9 and -3 by Apaf- 1 apoptosome and associated XIAP complexes. EMBO J. 2001;20:998-1009.

81. Galluzzi L, Kepp O, Kroemer GI. RIP kinases initiate programmed necrosis. J Mol Cell Biol. 2009;1:8-10.

82. Trichonas G, Murakami Y, Thanos A, et al. Receptor interacting protein kinases mediate retinal detachment-induced photoreceptor necrosis and compensate for inhibition of apoptosis. Proc Natl Acad Sci. 2010;107:21695-700.

83. He S, Wang L, Miao L, et al. Receptor interacting protein kinase3 determines cellular necrotic response to TNF-alpha. Cell. 2009;137:1100-11.

84. Meylan E, Tschopp J. The RIP kinases: crucial integrators of cellular stress. Trends Biochem Sci. 2005;30:151-9.

85. Festjens N, Vanden Berghe T, et al. RIP1, a kinase on the crossroads of a cell's decision to live or die. Cell Death Differ. 2007;14:400-10.

86. Lin Y, Devin A, Rodriguez Y, Liu ZG. Cleavage of the death domain kinase RIP by caspase-8 prompts TNF-induced apoptosis. Genes Dev. 1999;13:2514-26.

87. Zhang DW, Shao J, Lin J, et al. RIP3, an energy metabolism regulator that switches TNF-induced cell death from apoptosis to necrosis. Science. 2009;325:332-6.

88. Cho YS, Challa S, Moquin D, Genga R, Ray TD, et al. Phosphorylation-driven assembly of the RIP1-RIP3 complex regulates programmed necrosis and virus-induced inflammation. Cell. 2009;137:1112-23. 